\title{
Minimalistic thoracoscopic anterior spinal release in Scheuermann kyphosis
}

\author{
Eugenio Pompeo, MD, $\mathrm{PhD}$, Rome, Italy
}

\section{Fing}

Video clip is available online.

Scheuermann disease is the most common cause of thoracic kyphosis in adolescence. In advanced stages, the disease can cause severe kyphosis $\left(>70^{\circ}\right)$ and anterior wedging of thoracic vertebrae, necessitating anterior spinal release through a thoracotomy ${ }^{1}$ or video-assisted thoracoscopic surgery (VATS) followed by posterior spinal instrumentation and fusion for correction. ${ }^{2}$ I describe here a novel

From the Department of Thoracic Surgery, Policlinico Tor Vergata University, Rome, Italy.

Disclosures: Author has nothing to disclose with regard to commercial support.

Received for publication April 3, 2013; accepted for publication April 23, 2013; available ahead of print May 20, 2013.

Address for reprints: Eugenio Pompeo, MD, PhD, Sezione di patologia polmonare medica e chirurgica, Dipartimento di Biomedicina e Prevenzione, Policlinico Universitario Tor Vergata, Via Montpellier 1, 00133 Rome, Italy (E-mail: pompeo@ med.uniroma2.it).

J Thorac Cardiovasc Surg 2013;146:490-1

$0022-5223 / \$ 36.00$

Copyright (c) 2013 by The American Association for Thoracic Surgery

http://dx.doi.org/10.1016/j.jtcvs.2013.04.009 ultra minimally invasive (minimalistic) thoracoscopic anterior spinal release that allows excellent results in severe Scheuermann kyphosis.

\section{CLINICAL SUMMARY}

A 14-year-old girl with a diagnosis of severe Scheuermann kyphosis (Cobb angle, $83^{\circ}$ ) was admitted to Tor Vergata University (Rome, Italy) for surgical correction. After obtaining written, informed consent from the patient's parents, my team decided to perform VATS anterior spinal release with subsequent posterior instrumentation and fusion.

After placement of a nasogastric tube and intubation with a double-lumen tube for single-lung ventilation under general anesthesia, the patient was placed in the lateral leftsided decubitus position with $45^{\circ}$ semiprone rotation. Through a right-sided thoracoscopic access, three 7-mm flexible trocars were inserted: 1 for a $5-\mathrm{mm}, 30^{\circ}$-angled camera at the 10th intercostal space along the middle axillary line and 2 for instrumentation at the 10th and 7th intercostal spaces along the anterior axillary line.

Once the mediastinal pleura was incised, diskectomy was performed with dedicated thoracoscopic Kerrison forceps (MIASPAS; Aesculap, Inc, Center Valley, Pa). Performing

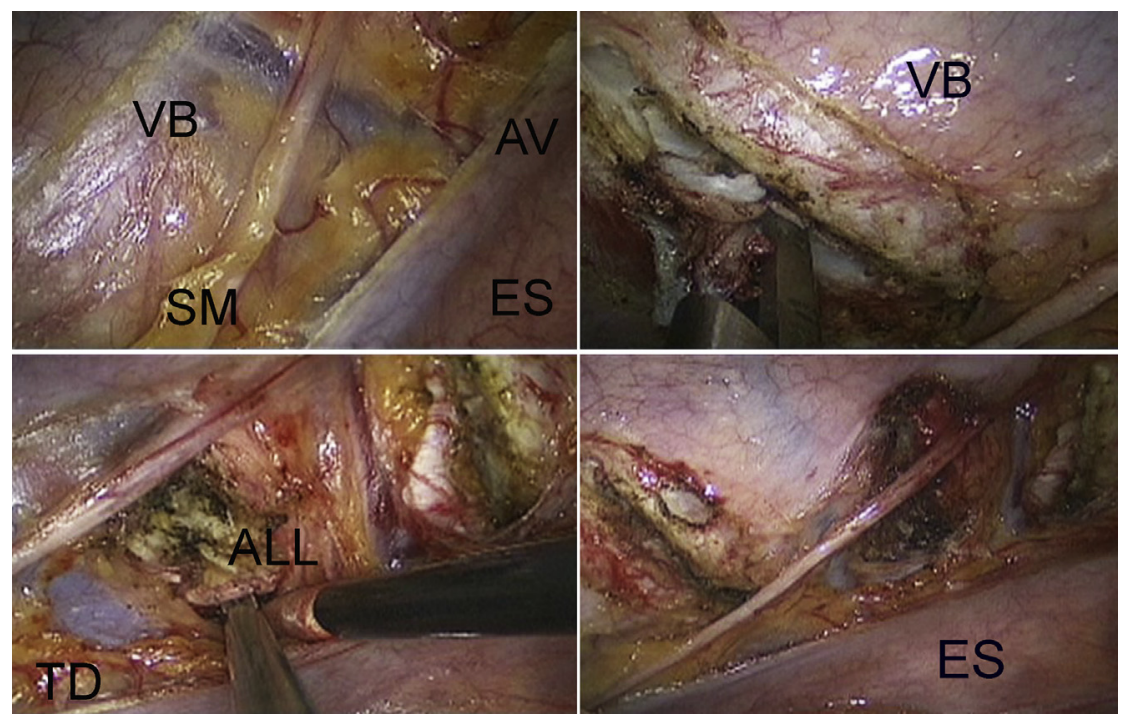

FIGURE 1. Intraoperative right thoracoscopic views of perivertebral anatomy. Upper left, The operative field is delimited by the esophagus (ES) and azygos vein $(A V)$ anteriorly and by the splanchnicus major nerve $(S M)$ running oblique to the T9 vertebral body $(V B)$. Upper right, After incision of the mediastinal pleura, complete diskectomy is performed first, with no division of segmental vessels. Lower left, Afterward, division of the anterior longitudinal ligament $(A L L)$ is carried out with minimal blunt dissection in the mediastinum, taking care to recognize and spare the thoracic duct (TD). Lower right, The final operative view shows the completed spinal release at the level of T8 through T10, with maximal sparing of anatomic structures in the posterior mediastinum. 
diskectomy as the first step facilitated exposure of the anterior longitudinal ligament from the inner side of the emptied disk spaces. This avoided the need for any sharp dissection anterior to the ligament. The ligament was then divided transversely at the level of the 3 most wedged and rigid disk spaces without use of electrically activated instruments. During this maneuver, mediastinal fatty tissue was gently displaced contralaterally with 5-mm cotton swabs. Segmental vessels were not divided, and attention was paid to recognize and spare the thoracic duct, the sympathetic nerves, and the azygos vein. Once the division of the ligament was complete, vertebral mobility was tested by rotating a Cobb elevator inside the released disk spaces. Finally, a chest tube was placed and the small skin incisions were sutured (Figure 1 and Video 1).

Posterior instrumentation and fusion were performed successfully 5 days later, and the patient was discharged uneventfully 10 days after the first procedure. Before discharge, chest radiography demonstrated a $100 \%$ correction of the hyperkyphosis (Cobb angle, $40^{\circ}$; Figure 2). At the last follow-up, 12 months after the operation, the patient is highly satisfied and has had no loss of correction.

Overall, I have used this minimalistic VATS approach in 4 cases of severe thoracic deformity. ${ }^{3}$ The overall correction rate averaged $75^{\circ}$, with no loss of correction at a median follow-up of 28 months.

\section{DISCUSSION}

To date, the only series of VATS anterior spinal release in Scheuermann kyphosis has been reported by Herrera-Soto and coworkers. ${ }^{2}$ Theoretical advantages of VATS include better cosmesis, magnified vision of and more direct approach to disk spaces, reduced blood loss, lower infection rate, and reduced thoracic pain, as well as shorter hospital stay. Disadvantages include a steep learning curve, the need for specialized equipment, loss of tactile feedback, and more difficult management of vascular complications. ${ }^{2}$

The original VATS approach that I propose is aimed at minimizing the overall invasiveness of the procedure. ${ }^{3}$ To accomplish this result, I recommend the use of 5-mm instruments passed through 2 intercostal spaces only to minimize both surgical trauma and postoperative pain and to improve cosmetic results. In addition, performing diskectomy as the first step facilitates the subsequent visualization of the anterior longitudinal ligament directly from the inner side of the emptied disk spaces and allows division of the ligament without sharp maneuvers carried out anterior to the ligament. The rationale of this minimalistic approach seems in accordance with the anatomic study of OuYang and Ding, ${ }^{4}$ who have recently hypothesized that by taking the intervertebral disk as a reference mark thoracoscopic spinal surgery might be safely performed without the need for

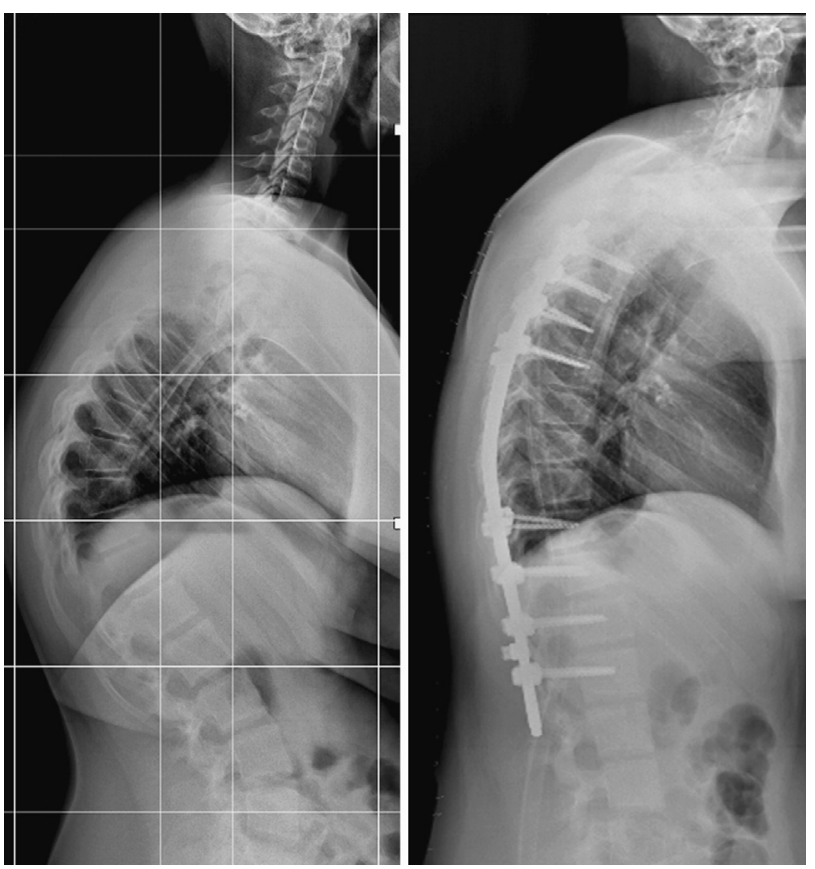

FIGURE 2. Left, Preoperative lateral radiograph showing significant thoracic kyphosis with a Cobb angle of $83^{\circ}$. Right, Postoperative radiograph demonstrating correction of the spinal deformity to $40^{\circ}$.

ligation of segmental arteries. This also avoids the risk of inadvertent damage to the Adamkiewicz artery, which nourishes the thoracolumbar segment of the spinal cord and has been found to originate in $91 \%$ of instances between the T8 and L1 levels. Another structure that needs to be clearly identified and spared during this procedure is the thoracic duct, which runs right sided and close to the anterior longitudinal ligament at this level.

Success of thoracic spine surgery requires multidisciplinary team cooperation, including dedicated thoracic and orthopedic surgeons.

In conclusion, this novel VATS anterior spinal release method achieved excellent results in severe Scheuermann kyphosis. Further investigation is warranted to confirm these preliminary findings.

\section{References}

1. Lim M, Green DW, Billinghurst JE, Huang RC, Rawlins BA, Widmann RF, et al Scheuermann kyphosis: safe and effective surgical treatment using multisegmental instrumentation. Spine (Phila Pa 1976). 2004;29:1789-94. Erratum in: Spine. 2004;29:2198.

2. Herrera-Soto JA, Parikh SN, Al-Sayyad MJ, Crawford AH. Experience with combined video-assisted thoracoscopic surgery (VATS) anterior spinal release and posterior spinal fusion in Scheuermann's kyphosis. Spine. 2005;19:2176-81.

3. Pompeo E, Mancini F, Ippolito E, Mineo TC. Videothoracoscopic approach to the spine in idiopathic scoliosis. Thorac Surg Clin. 2010;20:311-21.

4. OuYang H, Ding Z. Research of thoracolumbar spine lateral vascular anatomy and imaging. Folia Morphol (Warsz). 2010;69:128-33. 